\title{
Revisiting a Celebrated Bakerian Lecture
}

\author{
Salvatore Ganci \\ Studio di Catalogazione e Conservazione Strumenti Scientifici, Tromello, Italy \\ Email: museodellascienza.s.ga nci@gmail.com
}

How to cite this paper: Ganci, S. (2019)

Revisiting a Celebrated Bakerian Lecture. Optics and Photonics Journal, 9, 37-51. https://doi.org/10.4236/opj.2019.94005

Received: March 13, 2019

Accepted: April 25, 2019

Published: April 28, 2019

Copyright $\odot 2019$ by author(s) and Scientific Research Publishing Inc. This work is licensed under the Creative Commons Attribution International License (CC BY 4.0).

http://creativecommons.org/licenses/by/4.0/

\begin{abstract}
This paper revisits the optical contributions of Thomas Young to the theory of diffraction and, in particular, some experiments presented in his celebrated "Bakerian Lecture" of 1803. The major input to re-analyze some of Young's experiments came by a recent paper appeared on an instructional and pedagogical journal. Diffraction experiments of Thomas Young are here revisited with particular reference to the coherence requirements of the white light source to see and measure diffraction fringes by pins, and in general to consider the difficulties in working in a "camera obscura". If at the beginning of the nineteenth century, the experiments of Optics in a darkroom were considered "simple", however they needed rooms about 7 meters long, completely darkened out, with a window mostly exposed to south, a darkened window and a small hole practiced on a dark sheet applied to a hole in the window shutter. Often a heliostat should be placed outside and a mirror inside the room to direct the cone of sunlight. Thomas Young worked only with a mirror interior to the darkened room in order to have a cone of light horizontally directed and moving the mirror at each observation. Today, these requirements are not easy to implement. However, only a single measure by Thomas Young will be repeated under his own conditions with a "reduced darkroom" using internally blackened cardboard tubes.
\end{abstract}

\section{Keywords}

History of Physics, Diffraction Theories, Boundary Diffraction Wave Theory, Fresnel Diffraction

\section{Introduction}

"Any deviation of light rays from rectilinear paths which cannot be interpreted as reflection or refraction is called diffraction". This sentence opens the chap. 5 of one of the most classical books of Optics [1]. The observation of phenomenology related to diffraction requires some experimental conditions, such as a 
"light point source", openings and/or "small" obstacles that introduce a sharp discontinuity in the incident spherical wavefront that, at a great distance from the source, can be treated as a "plane wavefront".

These practical conditions were correctly recognized by Thomas Young starting from the 1802 papers [2] [3]. In the first of these papers, a first Boundary Theory of Diffraction was proposed and here it is found for the first time the Principle of Interference.

The major point attempted in this paper is an attentive reading of Thomas Young's contributions in order to particularly reproduce its own experimental conditions. We will see how, in the 1803 Bakerian Lecture [4], the requirement of coherence of the source using sunlight, in order to obtain diffraction fringes with fair readability, had to require a good adaptation of the eye to the dark and a challenge to one's own visual abilities.

\section{Historical Survey}

As pointed in the Sect. 1, the first "wave theory of diffraction" is found in the 1802 Young's paper [2]. The basic statement is the assumption of the periodic nature of the light and the interference principle, which is re-formulated with slight differences through all its papers on this matter [1] [2] [3] [4] [5]. Thomas Young assumed the wavefield through an aperture or an obstacle as the superposition between the unperturbed incident wavefront and an edge-diffraction wave arising at each point of the rim of the aperture or the obstacle. The origin of this edge-wave is confused. At the first time, the edge-wave is described as a kind of reflection on the edge by the incident wavefront; afterwards, in the same paper [2], Young considers diffraction as a refraction through an ether atmosphere surrounding the edge and having a density gradient in substantial agreement with the Newtonian description given by M. De Mairan [6]. The ether gradient hypothesis is not mentioned at all in the following paper [3], explicitly rejected in the following Bakerian Lecture [4] and ambiguously re-proposed in his "Lectures" [5] leaving diffraction without any theoretical support. A final observation on some difficulties that Thomas Young seems to have noticed appears in his latest paper on the subject [7] where ether is marginally considered as a necessary mean of propagation, and there is no mention of the analogy between light and sound, whereas the "interference principle" is again reiterated at the beginning of the paper. The full understanding of the resolving power of a diffraction grating as proportional to the number of lines cannot be unnoticed. It appears also noticeable how the mathematical language has completely changed. The style of the article reveals no emphasis, and no mention of the models of optical radiation. The term "diffraction" is the only one to be employed, while in the Bakerian Lecture [4] and in the Lectures [5] a promiscuous terminology is used (the Newtonian "inflection" and the provocative "diffraction").

The Boundary Diffraction Wave Theory seems to have been so natural that A. Fresnel also adopted the same Young's ideas in his first investigations without 
knowing Young's contributions [8]. His experiments about the influence of edge-parameters (material, cross-sectional geometry) made him change his mind about edge-diffraction wave approach, so that he developed his own theory of diffraction based on Huygens' concept of secondary wavelets [9]. A. Fresnel gave quantitative description of diffraction phenomena, but Huygens' Principle is not a "Theory". Therefore, Fresnel contributions to studies on diffraction received high consideration because observed phenomena were in good agreement with description provided by Fresnel. As a matter of facts, Young's diffraction Theory has been forgotten for many decades and Fresnel analysis was accepted and highly considered through the XIX century.

Also Fresnel analysis remained without any mathematical support until 1883 [10] when the diffracted wavefield was expressed as a solution-form of the wave-equation by superposition of spherical wavelets emitted by virtual sources on the plane of the aperture. Some years later, G. A. Maggi, showed how Kirchhoff integral formula could be converted into a line integral around a boundary line rim of an aperture or obstacle [11]. Maggi's contribution could not be considered as an explicit re-discovery of Young's ideas. Also, A. Rubinowicz, independently from Maggi's paper, explicitly re-discovered Young's ideas converting Kirchhoff integral formula into a line integral around the line $\Gamma$ boundary of the aperture in a "black" screen [12] [13] [14] with no approximation. Considering all the historical considerations on the Scalar Theory of Diffraction, it can be observed how two theories apparently so different, are at the end equivalent. In one of these it is emphasized that the diffraction is an effect of superposition of wavelets due to "sources" distributed in the aperture-plane, in the other it is emphasized that the diffraction arises from the overlap between the wave "geometrically cut" from the aperture and spherical wavelets coming from each point-sources on the edge of the aperture [15].

\section{Reading Thomas Young Paper}

Let us refer to 1804 paper [4] where experiments were more emphasized than theory. The paper is open with a sentence where T. Young claims: "I have found so simple and so demonstrative a proof of the general law of the interference of two portion of light...". Hence a first qualitative experiment is done. Experimental setup described appears very simple at his age. A small hole in a window-shutter covered with a thick card where a pin-hole was punched and a mirror out the window give a sunlight "point" source. A cone of diverging light passes the space through a table up to the opposite wall of the carefully obscured room. It appears evident that Thomas Young observes a Fresnel diffraction phenomenon (point source and a spherical wave). A fundamental observation then follows: the shadow of a rectilinear slip of card (breadth about $0.85 \mathrm{~mm}$ ) is surrounded by exterior fringes (similar to the fringes of a half-plane and a system of interior fringes of smaller dimensions) in parallel with the edge of the slip of card. Thomas Young claims these interior fringes differing in number, ac- 
cording to the distance at which the shadow is observed, but leaving the middle of the shadow always white. Historically his last fundamental observation was previously noticed by the Newtonian M. De Mairan [6] where a figure showing this phenomenology in the case of a rectilinear obstacle but also in the case of circular screen (the so called "Poisson Spot") was realized [16]. In both experiments, it is implicitly verified the spatial coherence requisite of the source along all the distances involved between the source (the hole in the window shutter) and the slip of card whose width is little less than $1 \mathrm{~mm}$. The "crucial" demonstration is the following: a few centimeters before the slip of card a sharp opaque screen moves towards one of the edge of the slip card and parallel to that edge. The exterior fringes disappear one by one and no perturbations are seen in the opposite and symmetric1lal exterior fringes. When the edge of the screen is touching the "geometrical" line from the screen's edge and the slip-edge, the interior fringes disappear. Thomas Young do not refers a change in approaching the edge of the slip card and infer that this portion of light "bending into-shadow" do not interfere with the other "portion of light" giving a disappearing of the interior fringes. T. Young claimed it to be "...the demonstrative proof of the general law of the interference". Curiously, a similar experiment was used later by Henry Brougham (a "natural" enemy of T. Young) at support of his "corpuscular" nature of light [17], and by G. Burniston Brown in his "new treatment of diffraction" [18].

In his Table I Obs. 9. $N$ and in Table II Obs. $3 N$, T. Young quotes two experiments in the third book of I. Newton [19] and in the Table III some measurements where T. Young point out better experimental setup used in all his observations. The distance from the source of light and the wall of the observation was $6.35 \mathrm{~m}$, the distance between the source and the aperture was $Z_{1}=3.18 \mathrm{~m}$ about even the rectilinear obstacle was $1 \approx 11 \mathrm{~mm}$ in width. Because the sun has an angular diameter $\theta \approx(1 / 2)^{\circ}$ the "geometrical diameter" of the sunbeam spot on the obstacle-edges was about $55 \mathrm{~mm}$. and consequently the "geometrical shadow" of the obstacle (not quoted by Young) were about $22 \mathrm{~mm}$, but Young refers his measurements to the external fringes and neglects the internal fringes. Of course, in order to see interference fringes with an acceptable degree of contrast the practical condition [19]:

$$
a \frac{D}{z_{1}} \ll \lambda
$$

must be verified, where in Equation (1) $a$ is the pin-hole diameter in the window-shutter, $z_{1}$ is the distance between the light source and the diffracting system, $D$ is the diameter of the light spot impinging on both edges of the diffracting system and $\lambda$ is the wavelength of the light. In white light, a "mean" wavelength $\lambda \approx 500 \mathrm{~nm}$ gives the magnitude order to considered verified Equation (1). In spite of the high frequency that the internal fringes should had o have, the visibility of them should have assumed a small hole-as light source-having diameter $\mathrm{a}<<0.15 \mathrm{~mm}$. 
In the quoted "Exper. 4." the distance $z_{1}$ between the "light point-source" and a wire whose width was $l \approx 2.1 \mathrm{~mm}$ reduced to $z_{1} \approx 0.8 \mathrm{~m}$ and the mean of the "geometrical shadow" observed on the opposite wall was claimed about $21 \mathrm{~mm}$. The measure is in agreement with a geometrical calculus on the similitude of the triangles isosceles S $I$ and $S s$ where $S$ is the vertex at the "point source" $l$ the width of the wire and $s$ the "breadth" of the shadow. Again, only the external fringes were considered and the coherence condition given by Equatiob (1), Young should have assumed a small hole-as light source-having diameter a $<<0.2 \mathrm{~mm}$.

\section{Revisiting Young's Experiments}

a) In the beginning of this investigation, an experimental apparatus similar to that used by Thomas Young would have been difficult to reproduce. Today the author has been unable to find a laboratory adapted as a darkroom in two physics departments. Indeed, it would have required a great Lab room, which they should have been south exposed and carefully darkened, a heliostat and a pinhole in a windows shutter. So, a "reduced camera obscura" was assembled using cardboard tubes of about $40 \mathrm{~mm}$ in diameter, carefully blackened with matt black paint in order to have a final tube long about $1.80 \mathrm{~m}$ well assembled on a wood support. One end of the tube was closed with thick blackened card and a central hole was made in an Aluminum foil ensured to the blackened card. The minimum hole diameter allowed was $0.35 \mathrm{~mm}$ (like a pinprick for diabetes diagnostic test). The sunlight, after reflection on a mirror, was sent into the hole acting as the source. A little screen of tracing paper was positioned near the end of the tube and a photographic camera was carefully assembled with the objective in contact with the end of the tube in order to focus on the shadow of a pin on the tracing-paper screen. A blackened pin (width $0.65 \mathrm{~mm}$ ) was placed at a distance $Z_{1} \approx 0.8 \mathrm{~m}$ from the point source in full agreement with the conditions used by Young in his last quoted experiment. In our experiment only the width of the wire was strongly reduced. Although the pin was less broad than that used by Thomas Young, the results were not satisfactory. Aside from undesired blurring caused by the vision of a fine-detail image on tracing paper, a single internal white fringe has been seen, even the external fringes appeared just recognizable as shown in Figure 1. If we apply Equation (1) to the wire used at a distance 0.8 $\mathrm{m}$ from the source, the "spatial coherence requirement" is very poorly verified. This attempt allows us to realize that when the eye is well accustomed to working with shadows using a faint cone of light in a completely darkened room, the recognition of interference fringes in white light can be a not entirely trivial enterprise. It can be inferred that in a darkroom the image quality is better and is not affected by blurring caused by vision through translucent paper. Young's pin where $2.11 \mathrm{~mm}$ in diameter, so, the coherence requirement should be satisfied by a "point source" having an amplitude $a<<0.2 \mathrm{~mm}$ with obvious problems in vision because the very low intensity of the light. 


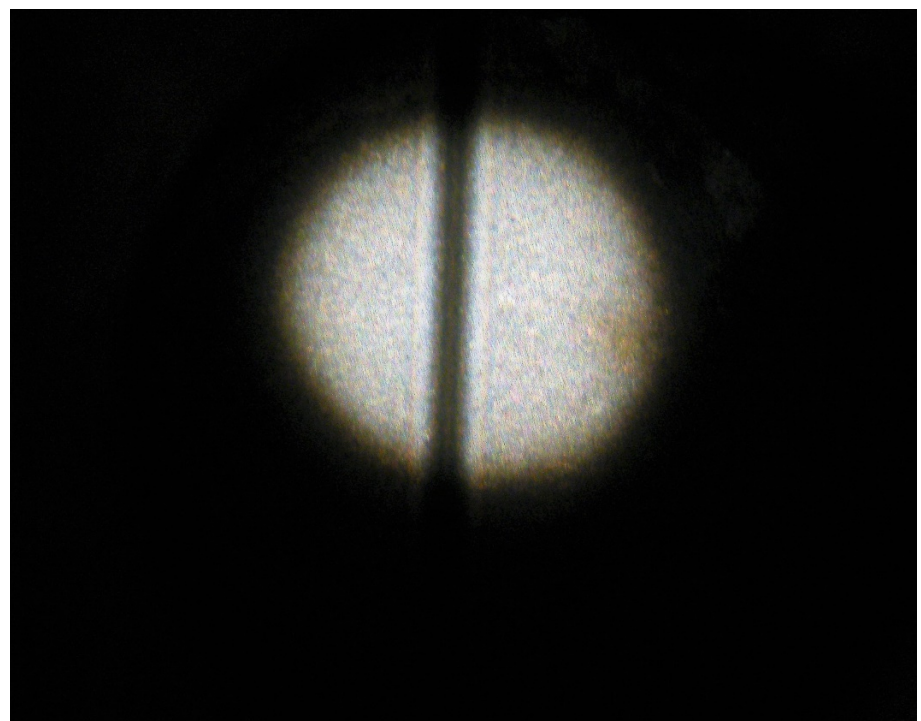

Figure 1 . The shadow of a pin in sunlight through a pin-hole (diameter $0.35 \mathrm{~mm}$ ) at a distance $z_{1} \approx 0.8 \mathrm{~m}$ viewed through a tracing paper that blurs some details. A light fringe at the center of the luminous area is (barely) recognizable at the center of two dark fringes. Not well defined the external fringes. As referred in the text, the spatial coherence requirements of the source are not fully verified. Photograph is unretouched.

b) Amore easy experimental attempt was carried out using a solid-state Laser emitting blue-violet light (nominal wavelength $\lambda \approx 405 \mathrm{~nm}$ ) with its terminal lens adjustable in order to have a cone of diverging light impinging on a blackened pin that can be positioned at different distances from the source. We can then obtain a divergent cone of light with the same angular divergence of that generated by a beam of sunlight through a small hole. The shadows, exterior and interior fringes, could be directly recorded on a sheet of glossy photographic paper ensured to the opposite wall at a distance about $3 \mathrm{~m}$ and using a front silvered mirror at a distance $6.3 \mathrm{~m}$ as in the Young's experiments. Figure 2 shows one of the more recorded images. To change the photo negative into a positive we use a quick procedure by finely scanning the recorded image and its conversion using an image processor software. Even the measurements of the inter-fringes distances can be made directly on the recorded image.

c) On the fringes internal to the shadow Thomas Young comments: “...differing in number, according to the distance at which the shadow was observed, but leaving the middle of the shadow always white". An experimental verification of this sentence shows that the crucial distance causing the increase of the fringes inside the shadow is only the distance source-diffracting object. In Figure 3(a) and Figure 3(b) it is shown the same shadow when the distance between the source and the pin (width of the pin $l=0.65 \mathrm{~mm}$ ) when the pin is respectively put at distances $0.4 \mathrm{~m}$ and $0.8 \mathrm{~m}$ from the source. Over the distance $0.8 \mathrm{~m}$ the shadow becomes narrower but the number of dark fringes with the central fringe always luminous remains unchanged. It was verified up to the limit of the 


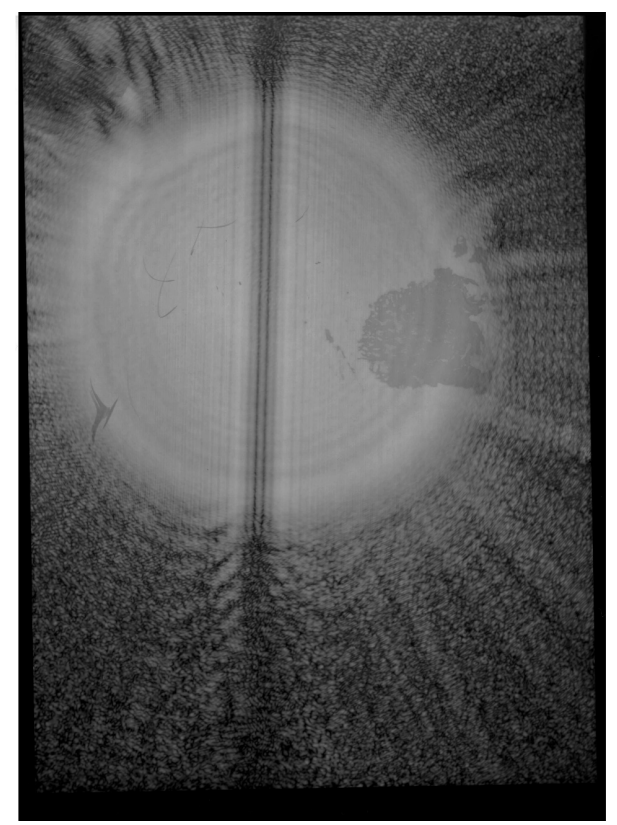

Figure 2. The shadow of the wire (width $0.65 \mathrm{~mm}$ ) obtained with a $50 \mathrm{~m}$ W solid-state blue laser (nominal wavelength $\lambda \approx 405 \mathrm{~nm}$ ) with its terminal lens adjustable in order to have a cone of diverging light impinging on a blackened wire that can be positioned at different distances from the source. A divergent cone of light with the angular divergence less great of that generated by a beam of sunlight through a small hole was employed, but the spatial coherence requirements of the source are better verified. Because the image (the negative of the image) is recorded directly on a photographic paper, quantitative measurements becomes possible on the image recorded, provided a greater photographic paper sheet and the same geometry used by Thomas Young. At a distance $Z_{1} \approx 0.8 \mathrm{~m}$ from the source and $z_{2} \approx 2.30 \mathrm{~m}$ between the wire and the observation plane, the interfringe is estimated about $2 \mathrm{~mm}$ using a caliper. A $10.5 \times 14.8 \mathrm{~cm}$ Ilford photographic paper $n^{\circ} 3$ is fixed on the observation plane; exposition time (manually accomplished moving an opaque screen) is about less than $1 \mathrm{~s}$. Development with Neutol 1:9 and Ilfosol rapid fixer bath.

naked eye visibility at about 2 meters between the source and the wire on a total distance $3.10 \mathrm{~m}$.

d) In the region of interest (e.g. the shadow of the wire), we have the superposition of two boundary waves arising at each edge. The idea of the "crucial" Young's experiment was the following: "If one of the boundary waves is suppressed, by means of a screen, then the other boundary wave does not superpose with the suppressed wave". Young's idea is fully correct, but, unfortunately, another boundary wave arises from the plane used for suppression. Its effect is visible when it is brought towards one of the wire-edge. The fringes outside the shadow come to disappear one at a time, and when the shadow of the screen is about to overlap the edge, the internal fringes first deform and then vanish while new fine fringes appear on the suppressing plane. When the overlap is completed the effect of the new "wave edge" introduced does not significantly disturb the system of opposite external fringes. 


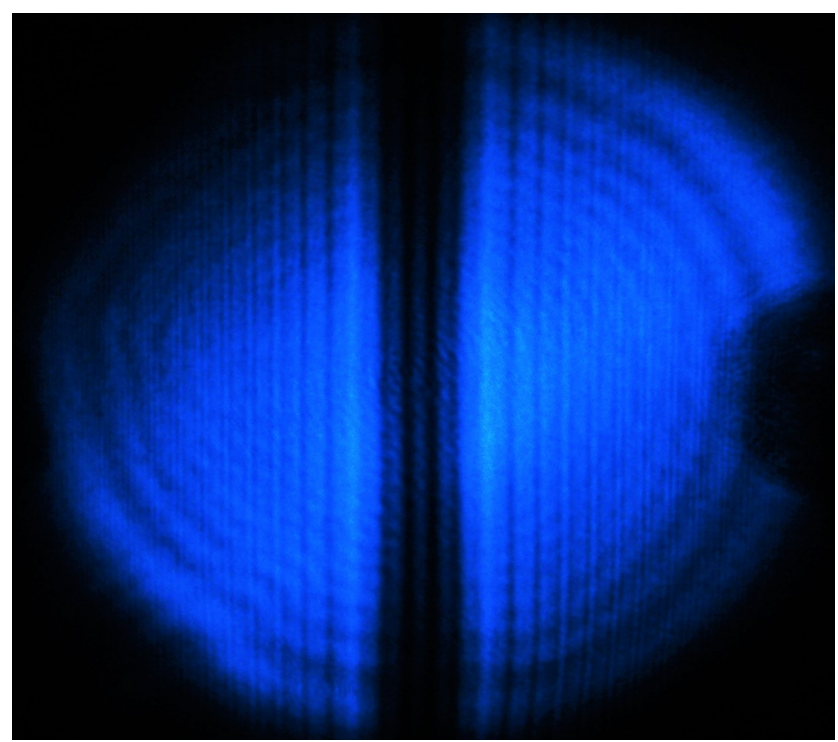

(a)

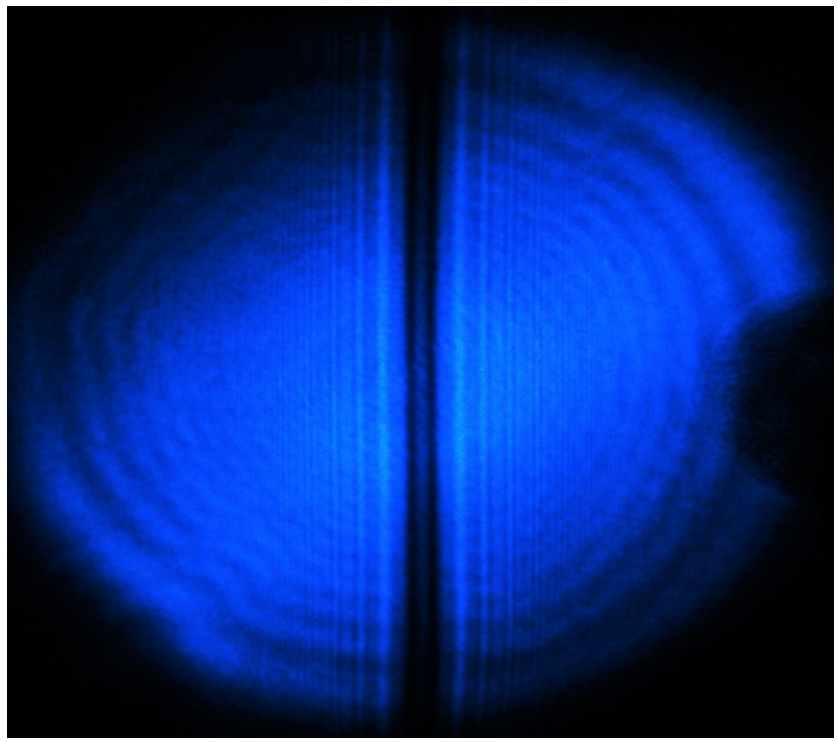

(b)

Figure 3. (a) Diffraction fringes of a wire having width $0.65 \mathrm{~mm}$ placed at $Z_{1}=0.22 \mathrm{~m} \pm 0.005 \mathrm{~m}$ from the source (a solid-state Laser) whose nominal wavelength is $\lambda \approx 405 \mathrm{~nm}$ viewed at a distance (after reflection on a front-silvered mirror) between source and screen $z_{1}+z_{2} \approx 6.54 \mathrm{~m} \pm 0.02 \mathrm{~m}$. The observation is made at about the same distance of the Young's experimental setup, but using a light source much easier to use. In the "geometrical shadow" there are two high-contrast black fringes and two black fringes having less contrast. The "geometrical shadowed area" directly estimated is $17 \mathrm{~mm} \pm 2 \mathrm{~mm}$. If the value $17 \mathrm{~mm}$ is inserted in the Equation (9) this equation is verified under a strong uncertainty of about $12 \%$ because of the difficulty to visually localize the "geometrical shadowed area". Different observers give slightly different values. The direct measurement of the two interior black interfringe using a caliper gives $4.0 \mathrm{~mm} \pm 0.2 \mathrm{~m}$; (b) Diffraction fringes of a wire having width $0.65 \mathrm{~mm}$ placed at $0.8 \mathrm{~m}$ from the same source in the previous figure caption. The number of interior black fringes is reduced from 4 to 2 . Overcoming the distance $0.8 \mathrm{~m}$ the pattern remains unchanged at least at the limit of naked eye visibility.

\section{Theoretical Analysis}

Boundary Diffraction Wave Theory (BDW) was the mathematical refinement of the Young's basic idea of the diffraction phenomenon. An attempt to formulate the theoretical analysis can be made using Maggi-Rubinowicz transformation of the Helmholtz-Kirchhoff integral formula into a line integral along the edge of the aperture or the obstacle.

$$
U(P)=\left\{\begin{array}{l}
U_{G}(P)+U_{B}(P), P \in J_{i} \\
U_{B}(P) ; P \in J_{s}
\end{array}\right.
$$

where $U_{G}(P)$ is the "geometrical" spherical wavefront propagating undisturbed through the diffracting object and $U_{B}(P)$ is the "boundary diffraction wave" arising at each point of the rim of the diffracting object. Similarly $J_{i}$ is the space region "geometrically illuminated" and $J_{S}$ is the "geometrically shadowed area". So, in the "geometrically illuminated" region, the wavefield is the superposition between the geometrical wavefront and a boundary wave; even in the geometrically shadowed region only the boundary wave acts. The general expression of the boundary diffraction wave in Equation (2) is the following:

$$
U_{B}(P)=\frac{1}{4 \pi} \oint_{r} \frac{\exp [i k(\rho+s)]}{\rho s} \frac{\cos (\boldsymbol{n}, \boldsymbol{s})}{1+\cos (\boldsymbol{\rho}, \boldsymbol{s})} \sin (\boldsymbol{\rho}, s) \mathrm{d} \boldsymbol{l}
$$


In Equation (3) we suppose a unit-amplitude spherical wave arising from a source S. So $k$ is the wave-number $2 \pi / \lambda$, where $\lambda$ is the wavelength, $\Gamma$ is the simple closed line rim of an aperture or an obstacle, $\exp (i k \rho) / \rho$ is the incident wavefield at a point Q of the edge $\Gamma$. The oriented distance $s$ is drawn from the observation point $\mathrm{P}$ and a point $\mathrm{Q}$ on the line $\Gamma, \boldsymbol{n}$ is a unit vector drawn, orthogonal to both the incident rays $\rho$ and to the line $\Gamma$. The general geometry of the problem is shown in Figure 4.

In our case the diffracting screen is rectilinear and with two edges. With reference to Figure 5 the geometry of the problem is shown. On an (undefined) rectilinear wire of width $l$, a unit-amplitude spherical monochromatic wavefield coming from a "point source" $S$, impinges symmetrically on both the edges of the wire (namely are equal $\rho$ and $\rho$ ). In order to apply the Maggi-Rubinowicz Transformation, the unit vector $\boldsymbol{n}$ is drawn, orthogonal to both the incident rays $\rho$ and $\rho^{\prime}$ (from the source $S$ at the points $Q$ and $Q^{\prime}$ of the edges) and to eachedge itself. Both $s_{1}$ and $s_{2}$ are the QP and Q'P vectors, which identify the observation point $P$ For simplicity, let us take $\mathrm{P}$ on the $x, y$ plane. Let be $U(P)$ the wavefield in a point $P$ of the $x, y$ plane. The formal solution to the problem of the interior fringes is the sum of two boundary waves, namely:

$$
U(P)=U_{B 1}(P)+U_{B 2}(P)
$$

Only the shadowed region is of interest here. Because the symmetry of the problem, $\rho=\rho^{\prime}$ (e.g. the source $S$ is on the $z$ axis); under the approximation $l<<$ $\rho$ and $l<<\mathrm{s}$, the unit vector $\boldsymbol{n}$ can be considered aligned with $X$-axis, namely only in the phase terms, $s_{1}$ and $s_{2}$ differ. Even under these stringent approximation two integrals such (3) in Equation (4) are not easy to solve. The method of the stationary phase (that falls when $\mathrm{P}$ approaches the "geometrical" boundary between illuminated and shadowed regions) gives a simple asymptotic solution to the problem. From the geometry in Figure 4, the phase is stationary when the incident rays at the edge and the oriented lines $s_{1}$ and $s_{2}$ are in the $y=0$ plane, thus, the problem becomes plane and its geometry is shown in Figure 5 where the wire width $l$ is drawn great compared with the "rays" length in order to shown the geometry and the approximations considered. Applying the method of the stationary phase [20] to an integral like (3) at each edge, the two boundary waves are:

$$
\begin{aligned}
U(P)= & U_{B 1}(P)+U_{B 2}(P) \\
= & \frac{1}{2 \pi} \frac{\exp (i k \rho)}{\rho}\left[\sqrt{\frac{\lambda}{s_{1}}} \frac{\cos \varphi_{1}}{1-\sin \varphi_{1}} \exp \left[i\left(k s_{1}+\pi / 4\right)\right]\right. \\
& \left.+\sqrt{\frac{\lambda}{s_{2}}} \frac{\cos \varphi_{2}}{1-\sin \varphi_{2}} \exp \left[i\left(k s_{2}+\pi / 4\right)\right]\right]
\end{aligned}
$$

The two expressions in Equation (5) have the asymptotic aspect of two cylindrical waves with angular factors that becomes infinity when $s_{1}$ or $s_{2}$ are aligned with the correspondent rays $\rho$ on the $y=0$ plane, as shown in Figure 6. The angles $\varphi_{1}$ and $\varphi_{2}$ identify the directions of the segments oriented from $\mathrm{P}$ to the points of the edge in the two-dimensional problem. So, strictly speaking 


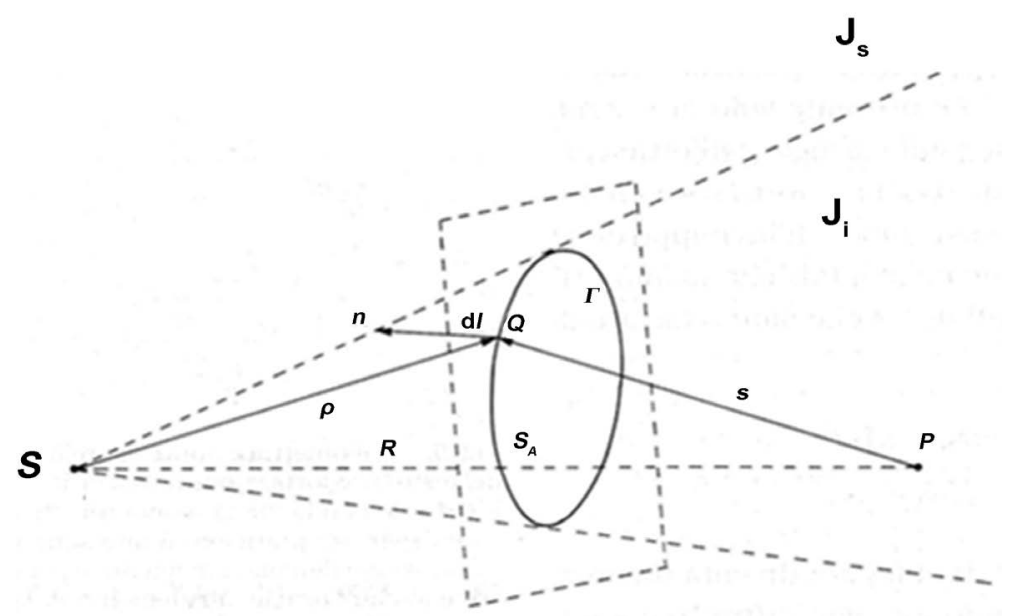

Figure 4. The general geometry of the Maggi-Rubinowicz transformation. Case of an aperture $S_{A}$ having the boundary the simple line $\Gamma$ on an opaque screen. The unit vector $\boldsymbol{n}$ is orthogonal to both the incident ray $\boldsymbol{\rho}$ and to the line $\Gamma$ at the point $Q . R$ represent the ray of the "geometrical wave" going in the illuminated region $J_{i}$ from the source $\mathrm{S}$ to the observation point $P$.

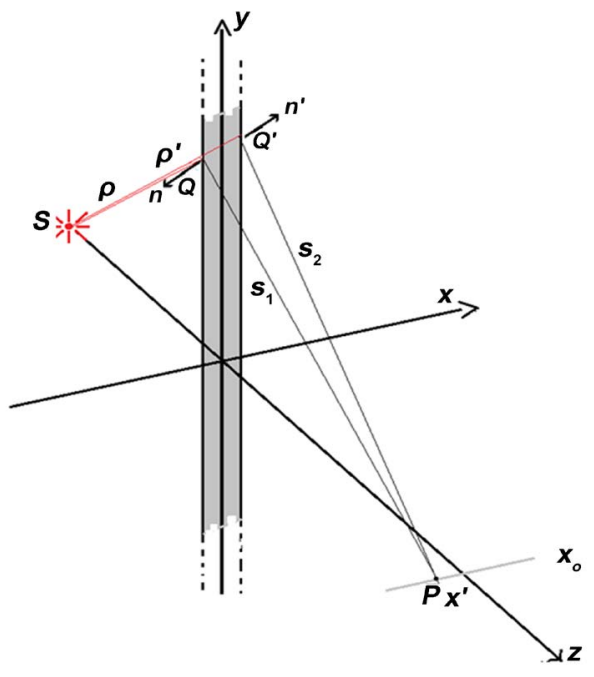

Figure 5. The Maggi-Rubinowicz Transformation applied to the diffraction of a rectilinear wire of width 1 . For the sake of simplicity in calculus, the light source $S$ is on $Z$-axis. The observation point $P$ is in the plane $x, Z$ on an axis $x_{o}$ parallel to $x$-axis. The strip has the $z$-axis as symmetry axis, so $\rho=\rho$. The unit vectors $n$ and $\boldsymbol{n}^{\prime}$ are both orthogonal to the edge of the wire and to the rays $\rho$ and $\rho$ 'respectively.

Equation (5) holds only near the center of the wire where the approximation $I$ $<<s_{1}, l<<s_{2}$, holds. The distances $s_{1}$ and $s_{2}$ are:

$$
\begin{gathered}
s_{1}=\sqrt{z_{2}^{2}+\left(l_{o} / 2-x_{o}\right)^{2}} ; s_{2}=\sqrt{z_{2}^{2}+\left(l_{o} / 2+x_{o}\right)^{2}} \\
s_{1} \approx z_{2}\left[1+\left(\frac{l_{o} / 2-x_{o}}{2 z_{2}}\right)^{2}\right] ; s_{2} \approx z_{2}\left[1+\left(\frac{l_{o} / 2+x_{o}}{2 z_{2}}\right)^{2}\right]
\end{gathered}
$$




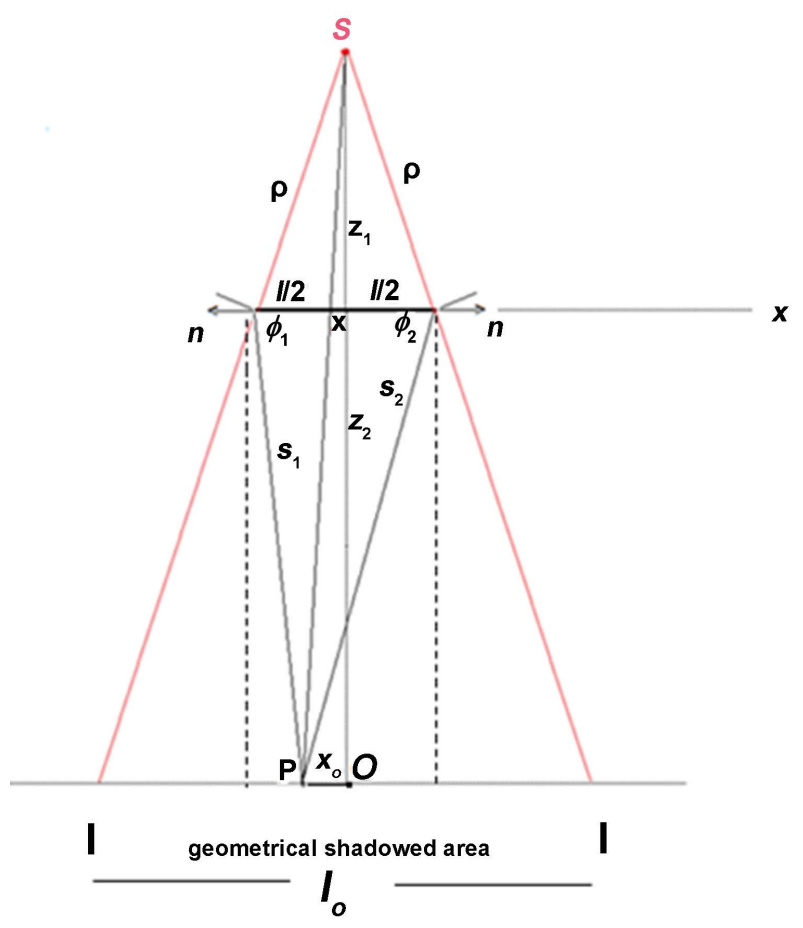

Figure 6. Applying the method of the stationary phase, the problem becomes two-dimensional. The asymptotic approximation introduced holds in a region of the geometrical shadow where the observation point $\mathrm{P}$ lies far from the boundary of the "geometrical shadowed area". Under the approximation $l<\rho \rho$, the unit normal vector $\boldsymbol{n}$ (that is orthogonal both to the incident ray at the edge and the edge itself) can be considered aligned with $x$-axis. Namely, a quasi-plane wave approximation is considered. No hypotheses are put on the distances between the diffracting wire and the observation point P. Between the wire-width and the "geometrical shadowed area" holds the proportion $1: z_{1}=I_{o}\left(z_{1}+z_{2}\right)$ where a line from the source $\mathrm{S}$ to the point $\mathrm{P}$ cut a segment $x$ on the wire to respect its center and a segment $x_{o}$ from the center of symmetry of the "geometrical shadowed area" on the observation plane.

where the binomial approximation of the square root was done. Under this special approximation, disregarding both amplitude and phase factor in Equation (5), the simple calculus of the optical path difference $\Delta s=\left|s_{2}-s_{1}\right|$ gives the maxima and minima inside the "geometrical shadowed area":

$$
\Delta s=\left|s_{2}-s_{1}\right|=\frac{2 l_{o} x_{o}}{z_{2}}
$$

where $s_{1}$ and $s_{2}$ are the distances between the observation point and the edges of the wire having width $I$ and the $X$-coordinate from the center of the wire is the intersection of a line drawn from the source $S$ and the observation point $P$. With reference to Figure 6, in Equation (8) the $x_{0}$-variable is the distance from the center $\mathrm{O}$ of the "geometrical shadowed area" of width $l_{0}, k$ is the wavenumber and $z_{2}$ is the distance from the slit and the observation plane. From the geometry of Figure 6 the below proportionalities follow: 


$$
z_{1}: x=\left(z_{1}+z_{2}\right): x_{o} ; z_{1}: l=\left(z_{1}+z_{2}\right): l_{o}
$$

Under the stringent approximations introduced, a simple inspection of Equation(8) with the relations in Equation (9) allow us an evaluation of the "geometrical shadowed area" under the condition $z_{1}+z_{2}$ fixed, and shows that the interfringe (considered on the observation plane) is dependent on both distance $Z_{1}$ and $Z_{2}$. The "quasi-plane" wave approximation simplified the calculus of a couple of integrals such as in Equation (3). Let us consider the $x_{0}$ coordinate, in the image-plane as the "geometrical" projection of the $x$ coordinate behind the diffracting wire, namely a distant source $S$. As a special case, we can consider the situation when the number of black interference fringes contouring the central luminous line is reduced to two. The condition that must be verified is that the interfringe distance between two dark lines is less than $l_{0}$. Moreover, the distance between two minima is estimated $2 \mathrm{~mm} \pm 0.5 \mathrm{~mm}$ and the "geometrical shadow" estimated in $3 \mathrm{~mm} \pm 0.5 \mathrm{~mm}$ in substantial agreement with the above theory. With reflection on a front-silvered mirror the pattern is brought at a distance source-screen $6.5 \mathrm{~m}$, about the same experimental situation of T. Young but not particular advantages were find. In the major part of the experiments a wavelength about $405 \mathrm{~nm}$ was used. It follows from (8) for a slit of width $0.65 \mathrm{~mm}$, a fixed distance $Z_{1}+Z_{2}=3.1 \mathrm{~m}$ (or $Z_{1}+Z_{2}=6.54 \pm 0.03 \mathrm{~m}$ after reflection into a front silvered mirror), a pattern having only two first minima contouring the central maximum is obtained at $z_{1} \geq 0.8 \mathrm{~m}$.

In the end, a "critical" experiment performed by the Author is leaved to the Reader's consideration. A flat and blackened wire (i.e. $0.65 \mathrm{~mm}$ in width) is mounted between two crossed linear polarizers. You must ensure a mounting of the polarizers' edges be carefully inside the wire. Light from a divergent beam of a solid-state laser is sent on this system and the diffraction fringes observed on a screen. Even when each system of external fringes appears unperturbed, the internal fringes (well visible without the polarizers) are completely suppressed. Regarding the diffraction as interference of the lights coming from the discontinuity in the wavefront caused by the wire's edges, now the light coming from the right edge no longer overlaps the light coming from the left edge because the polarizers are crossed. The overlap between the light on the left edge does not significantly affect the external fringes that are formed on the right and vice-versa. Obviously the above word "critical" is provoking, because Young's theory of the boundary wave and the Fresnel's theory of diffraction founded on Huygens principle are fully equivalent because they derive from the same integral formula.

\section{Conclusions}

An attentive critical reading of Thomas Young experiments presents some perplexities because, in general, Thomas Young is not always conscious of the more important consequences of its own measurements. Indeed, after some fundamental observations at the beginning of its own paper, he "switches off" its 


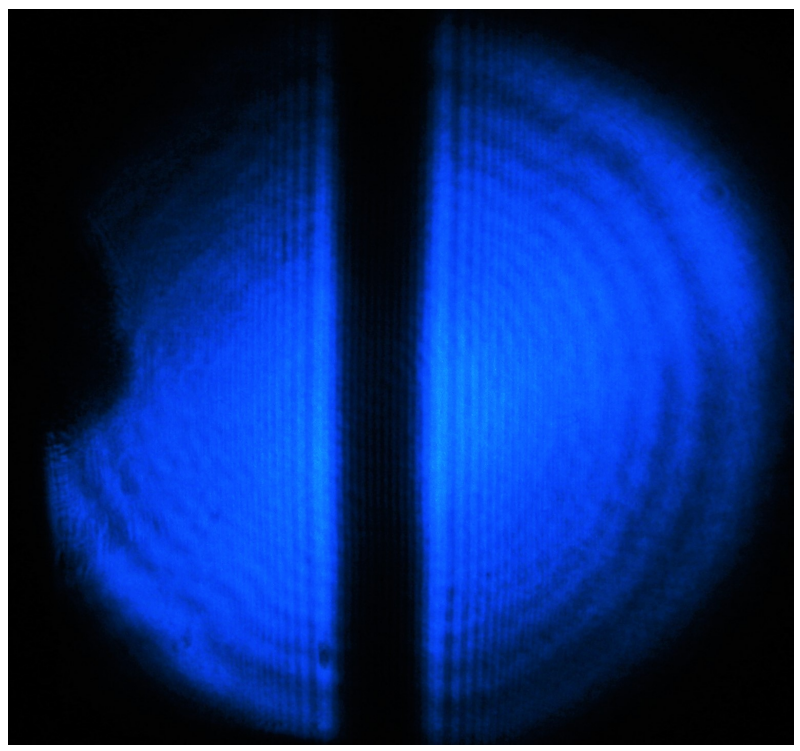

Figure 7. A very fine system of fringes viewed in about the same situation of Young's experimental work. Here the source is monochromatic and spatial coherence is ensured. The wire is at a distance $0.8 \mathrm{~m}$ from the Laser and the distance $Z_{1}+z_{2}$ is about the same distance of Young "obscured room". Only the divergence of the beam is slightly greater than the sunbeam of Young's experiment.

attention from the interior fringes, to the exterior fringes, the only fringes considered by Isaac Newton. Therefore, he is more concerned with the comparison of the position of the fringes outside the shadow with Newton's observations and measurements. Table III exper. 4 leaves a perplexity: IF the "breadth of the wire" is $0.083 \mathrm{inch}$, (namely, about $2.1 \mathrm{~mm}$ ) at a distance from the aperture (the source of light) of about 32" (about $0.8 \mathrm{~m}$ ), THEN the pattern of internal fringes is just seen using the divergent beam of the Laser used at the same distance as in Young's experiment. At a distance of $0.8 \mathrm{~m}$, the request for spatial coherence of the source (Equation (1) evaluated at a mean wavelength $\lambda \approx 500 \mathrm{~nm}$ ) would have required a small hole with a diameter of less than $0.2 \mathrm{~mm}$ as previously evaluated. Figure 1 taken at the same distance from a greater source of $0.35 \mathrm{~mm}$ in diameter shows the low resolution of the central maximum in the shadow even the image viewed through the trans-lucid paper inevitably is blurred. In the end, Figure 7 shows a very fine system of interior fringes using a wire of width 2 $\mathrm{mm}$ at the similar experimental situation of T. Young's work, but in coherent light. In this paper:

a) The coherence requirements in Young's experiment were analyzed in detail;

b) In an attempt to work in a "reduced camera obscura" we have "touched with hand" as at a distance of about one meter from the source, the requirement of spatial coherence of the source becomes very stringent;

c) A case of Fresnel Diffraction correctly describes what really Thomas Young 
observed [21];

d) BDW theory, historically starting from Young's point of view of diffraction phenomena, is applied to revisiting Young's experiments with good agreement between theory and experiment. It is always with great admiration and humility if today we revisit the experiments of Optics made throughout the nineteenth century taking into account the practical difficulties of an observer working in an "obscured-room".

\section{Acknowledgements}

The linguistic revision of Ms. Karen Rossi is acknowledged with sincere thanks. Sincere thanks to Alessio Ganci for linguistic revision of the revised manuscript. Thanks to my former student and friend Daniele Lagomarsino Oneto at Université Nice-Sophia Antipolis for a critical reading of the manuscript.

\section{Conflicts of Interest}

The author declares no conflicts of interest regarding the publication of this paper.

\section{References}

[1] Sommerfeld, A. (1954) Optics. Translated by Laporte, O. and Moldauer, P.A., Academic Press, New York, 179.

[2] Young, T. (1802) On the Theory of Light and Colours. Philosophical Transactions of the Royal Society of London, 92, 12-48. https://doi.org/10.1098/rstl.1802.0004

[3] Young, T. (1802) An Account of Some Cases of the Production of Colours Not Hitherto Described. Philosophical Transactions of the Royal Society of London, 92 , 387-497. https://doi.org/10.1098/rstl.1802.0016

[4] Young, T. (1804) I. The Bakerian Lecture. Experiments and Calculations Relative to Physical Optics. Philosophical Transactions of the Royal Society of London, 94, 1-16. https://doi.org/10.1098/rstl.1804.0001

[5] Young, T. (1807) A Course Lectures on Natural Philosophy and the Mechanical Arts. Taylor and Walton, London.

[6] De Mairan, M. (1760) Troisième Partie des Recherches Physico-Mathématiques sur la Réflexion des Corps. Mémoires de I Académie Royale des Sciences, 5, 19-42.

[7] Young, T. (1829) Theory of Colours Observed in the Experiments of Fraunhofer. The Edinburgh Journal of Science, 1, 112-116.

[8] Fresnel, A. (1866) Oeuvres Complètesd' Agustin Fresnel. Imprimerie Imperiale, Paris, Tome 1.

[9] Huygens, C. (1690) Tritè de la Lumière. Peter van der Aa, Leiden.

[10] Kirchhoff, G. (1883) Zur Theorie der Lichtstrahlen. Annalen der Physik, 254, 663-695. https://doi.org/10.1002/andp.18832540409

[11] Maggi, G.A. (1888) Sulla propagazione libera e perturbata delle onde luminose in un ezzo isotropo. Annals of Mathematics, 16, 21-48.

[12] Rubinowicz, A. (1917) Die Beugungswelle in der Kirchoffschentheorie der beugungerscheinungen. Annals of Physics, 53, 257-278.

https://doi.org/10.1002/andp.19173581202 
[13] Rubinowicz, A. (1924) Zur Kirchoffschen Beugungstheorie. Annals of Physics, 73, 339-364. https://doi.org/10.1002/andp.19243780504

[14] Rubinowicz, A. (1966) Die Beugungswelle in der Kirchhofscen Theorie der Beugung. 2nd Edition, Springer, Berlin.

[15] Ganci, S. (1997) Boundary Diffraction Wave Theory from Rectilinear Apertures. European Journal of Physics, 18, 229-235. https://doi.org/10.1088/0143-0807/18/3/018

[16] Ganci, S. (2013) Was Poisson Spot an Intentional Discovery? Optik, 124, 3906-3908. https://doi.org/10.1016/j.ijleo.2012.11.017

[17] Billet, F. (1858) Traitéd' Optique Physique, Tome 1. Imprimerie de Mallet-Bschelier, Paris, 537-538.

[18] Burniston Brown, G. (1963) A New Treatment of Diffraction. Contemporary Physics, 5, 15-27. https://doi.org/10.1080/00107516308205835

[19] Mayer-Arendt, J.R. (1972) Introduction to Classical and Modern Optics. Prentice-Hall, Englewood Cliffs, Chap. 2.6.

[20] Erdélyi, A. (1956) Asymptotic Expansion. Dover, New York, 51-56.

[21] Sheider, W. (1986) Bringing One of the Great Moments of Science to the Classroom. The Physics Teacher, 24, 217-219. https://doi.org/10.1119/1.2341987 